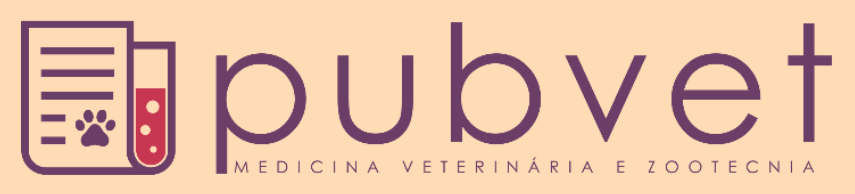

https://doi.org/10.31533/pubvet.v13n5a328.20-28

\title{
Fatores anti-nutricionais de importância na nutrição animal: Estratégias de diminuição dos efeitos anti-nutricionais
}

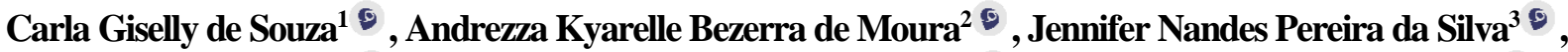

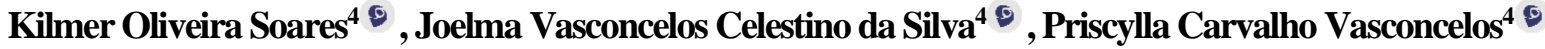 \\ ${ }^{1}$ Pós Doutoranda do Programa de Pós-Graduação em Zootecnia, Universidade Federal de Pernambuco, Brasil. \\ ${ }^{2}$ Professora IFPA (Instituto Federal do Pará) Campus Breves. \\ ${ }^{3}$ Mestrando(a) do Programa de Pós-Graduação em Zootecnia, Universidade Federal da Paraíba. Brasil- PB, Brasil \\ ${ }^{4}$ Doutorando (a) do Programa de Pós-Graduação em Zootecnia, Universidade Federal da Paraíba - PB, Brasil \\ *Autor para correspondência, e-mail: carlaxlsouza@yahoo.com.br
}

\begin{abstract}
Resumo: Os metabolitos secundários também conhecidos como fatores anti-nutricionais, nada mais são do que componentes naturais responsáveis por exercerem funções fisiológicas nos vegetais. São substâncias que, mesmo em estado vestigial, reduzem ou impedem a utilização de um elemento nutritivo, seja no nível digestivo, ou no nível metabólico. Entretanto devido a relevante importância na nutrição animal que alguns ingredientes exercem, estes precisam passar por um processo químico ou físico para viabilizar seu uso na nutrição animal, sem, entretanto, ter que "sofrer" com efeitos adversos do consumo excessivo de determinados compostos secundários. Deste modo, a presente revisão tem como objetivo elucidar sobre as estratégias para diminuição dos efeitos inerentes aos fatores antinutricionais na dieta dos animais. $\mathrm{O}$ tratamento térmico, extrusão, peletização e moagem são métodos muito utilizados para redução e/ ou inativação de substâncias indesejáveis em alimentos. Além disso, alguns animais naturalmente e evolutivamente desenvolveram estratégias para conviver com estes fatores.
\end{abstract}

Palavras chave: Cocção, prolinas, tratamento térmico

\section{Antinutritional factors of importance in animal nutrition: Strategies for decreasing anti-nutritional effects}

\begin{abstract}
Secondary metabolites also known as anti-nutritional factors are nothing more than natural components responsible for exerting physiological functions in plants. They are substances that, even in vestigial state, reduce or prevent the use of a nutritive element, either at the digestive level, or at the metabolic level. However due to the important importance in animal nutrition that some ingredients exert, they need to undergo a chemical or physical process to make it feasible to use in animal nutrition, without, however, having to "suffer" from the adverse effects of excessive consumption of certain secondary compounds. Thus, the present review aims to elucidate the strategies to reduce the effects inherent to the antinutritional factors in the animals' diet. Heat treatment, extrusion, pelletizing and milling are widely used methods for reducing and / or inactivating undesirable substances in food. In addition, some animals naturally and evolutionarily have developed strategies to cope with these factors.
\end{abstract}

Key words: Cooking, prolines, heat treatment 


\title{
Factores antinutricionales de importancia en la nutrición animal: Estrategias de disminución de los efectos antinutricionales
}

\begin{abstract}
Resumen. Los metabolitos secundarios también conocidos como factores antinutricionales, no son más que componentes naturales responsables de ejercer funciones fisiológicas en los vegetales. Son sustancias que, incluso en estado vestigial, reducen o impiden la utilización de un elemento nutritivo, ya sea en el nivel digestivo, o en el nivel metabólico. Sin embargo, debido a la relevante importancia en la nutrición animal que algunos ingredientes ejercen, éstos necesitan pasar por un proceso químico o físico para viabilizar su uso en la nutrición animal, sin, tener que "sufrir" con efectos adversos del consumo excesivo de determinados compuestos secundarios. De este modo, la presente revisión tiene como objetivo elucidar sobre las estrategias para disminuir los efectos inherentes a los factores antinutricionales en la dieta de los animales. El tratamiento térmico, extrusión, peletización y molienda son métodos muy utilizados para la reducción y / o inactivación de sustancias indeseables en alimentos. Además, algunos animales natural y evolutivamente desarrollaron estrategias para convivir con estos factores.
\end{abstract}

Palabras clave: Cocción, prolinas, tratamiento térmico

\section{Introdução}

Os fatores anti-nutricionais são componentes naturais responsáveis por exercerem funções fisiológicas nos vegetais (Francis et al., 2001). Isso não necessariamente quer dizer que eles não poderiam ter efeitos deletérios em humanos. Entretanto, com as evidências que atualmente temos disponíveis, não é possível afirmar que, nas quantidades normalmente ingeridas, seu consumo seria capaz de causar problemas. Ao contrário, é bem possível que eles tragam mais benefícios do que prejuízos, e, até por isso, há muito tempo sugere-se que o termo "anti-nutricional" seja revisto (Harborne, 1999).

Os fatores anti-nutricionais propriamente ditos são substâncias que, mesmo em estado vestigial, reduzem ou impedem totalmente a utilização de um elemento nutritivo, seja no nível digestivo, seja no nível metabólico (Harborne, 1999). Estes fatores anti-nutricionais, em sua maioria são metabólitos secundários. Estes metabólitos secundários das plantas podem ser definidos como compostos que não têm papel reconhecido na manutenção de processos vitais fundamentais (crescimento, desenvolvimento e reprodução) que os sintetizam, em contrapartida, têm um papel importante na interação da planta com seu meio ambiente. A produção destes compostos é frequentemente baixa (menos de $1 \%$ de peso seco) e depende muito do estágio fisiológico e de desenvolvimento da planta (Namdeo, 2007). Os fatores antinutricionais foram importantes, em um contexto evolutivo, pelos seguintes motivos: para "atrapalhar" a digestão dos animais que ingerissem, por exemplo, as sementes dos vegetais. Ou seja, para que o embrião das plantas pudesse sobreviver ao trato gastrointestinal do predador. Para causar efeitos gastrointestinais adversos nos predadores, evitando que as plantas fossem consumidas numa próxima ocasião.

Na tentativa de melhorar a digestibilidade dos alimentos e o desempenho dos ruminantes tem-se aplicado aos alimentos vários tipos de processamentos, podendo ser físicos ou químicos, onde atuam quebrando as barreiras físicas que impedem o acesso dos microrganismos ruminam aos alimentos, e aumentando a área de superfície dos grãos, reduzindo a interação da matriz proteica dos grânulos de amido (Kumar \& Sharma, 2017). Neste sentido, pré-tratamentos convencionais podem ser aplicados como: tratamento térmico, aplicação de enzima, imersão, brotação/germinação, irradiação e fermentação (Nikmaram et al., 2018) descascamento, maceração e cozimento. Além de moagem, extrusão, micronização, tostagem, peletização, laminação, floculação, explosão, cozimento e outros (Kumar \& Sharma, 2017). Outros métodos utilizados no processamento de alimentos também podem reduzir as concentrações dos fatores anti-nutricionais, tais como: a adição de água ao alimento, maceração na presença de sulfitos, trituração, descortiçamento de grãos, atmosfera controlada $\left(\mathrm{N}_{2}\right.$, etileno e etanol absoluto), tratamento enzimático, alta pressão hidrostática, dentre outros (Benevides et al., 2011). Segundo Owens et al. (1986) o aumento progressivo do processamento favoreceram a digestão ruminal. 
Deste modo, devido a relevante importância na nutrição animal que alguns ingredientes exercem, estes precisam realmente passar por um processo químico ou físico para viabilizar seu uso na nutrição animal, sem, entretanto, ter que "sofrer" com os consequentes efeitos adversos do consumo excessivo de determinados compostos secundários. Com isso, a presente revisão tem como objetivo elucidar sobre as principais estratégias para diminuição dos efeitos inerentes aos fatores anti-nutricionais na dieta dos animais.

\section{Tratamentos estratégicos para a diminuição dos efeitos dos fatores anti-nutricionais}

\section{Moagem}

Reis (2006), submetendo cultivares de milho a diferentes granulometrias, processado grosso (12 $\mathrm{mm}$ ), médio (10 $\mathrm{mm}$ ) e fino (oito $\mathrm{mm}$ ), observou que quanto menor a granulometria, maior a degradabilidade da matéria seca. Souza et al. (2010) estudaram dois híbridos de sorgo (com tanino e sem tanino), sob três granulometrias (inteiro, moído grosso $(6 \mathrm{~mm})$ e moído fino $(4 \mathrm{~mm})$, e observou que a moagem dos grãos de sorgo proporcionou aumento da degradabilidade ruminal e efetiva da matéria seca, proteína bruta e amido. Além disso, a presença do tanino no grão de sorgo não alterou a degradabilidade da matéria seca.

\section{Cozimento}

O tratamento térmico, dentre outras funções, é um método muito utilizado para redução e/ ou inativação de substâncias indesejáveis em alimentos (Benevides et al., 2011). Entretanto, cozinhar a alta temperatura e por muito tempo pode causar alterações físico-químicas indesejáveis nas proteínas, amido e a perda de nutrientes essenciais (vitaminas, aminoácidos) e outros valiosos constituintes sensíveis ao calor destas leguminosas (Benevides et al., 2011; Nikmaram et al., 2018).

Delfino \& Canniatti-Brazaca (2010) mediram a capacidade de hidratação e o tempo de cocção antes e após o armazenamento e avaliaram os teores de taninos em feijão comum, cultivar Pérola, e a interferência desses compostos na digestibilidade de proteínas. Estes parâmetros foram avaliados em feijões recém-colhidos e armazenados durante 3 e 6 meses, antes e após cocção. Para a cocção, o feijão foi lavado, deixado sob maceração por 12 horas e, posteriormente, cozido em autoclave por 10 minutos a $121^{\circ} \mathrm{C}$, tabela 1 .

Tabela 1. Teor de Tanino \%mEq de catequina (matéria seca) em feijão cru e cozido, e em diferentes tempos de armazenamento

\begin{tabular}{lccc}
\hline & Recém-colhido & Armazenado 3 meses & Armazenado 6 meses \\
\hline Cru & $1,13 \pm 0,00^{\mathrm{a} 2 \mathrm{~A} 3}$ & $1,10 \pm 0,00^{\mathrm{aA}}$ & $0,90 \pm 0,00^{\mathrm{bA}}$ \\
Cozido & $0,74 \pm 0,00^{\mathrm{aB}}$ & $0,62 \pm 0,00^{\mathrm{bB}}$ & $0,23 \pm 0,00^{\mathrm{cB}}$ \\
\hline
\end{tabular}

${ }^{1}$ Média de três repetições \pm desvio padrão; ${ }^{2}$ Média com letras maiúscula(s) deferente(s) na vertical diferem significativamente $(\mathrm{p} \leq 0,05) ;{ }^{3}$ Média com letras minúscula(s) diferente(s) na horizontal diferem significativamente ( $\left.\mathrm{p} \leq 0,05\right)$. Fonte: Delfino \& CanniattiBrazaca (2010).

Delfino \& Canniatti-Brazaca (2010) relatam que grande parte dos taninos é transportada por lixiviação à água de maceração, como pode ser observado pelos valores obtidos após cocção dos grãos, além disso, o aumento da digestibilidade nas amostras cozidas provavelmente se dá pela eliminação de fatores anti-nutricionais, cuja complexação com as proteínas impede a ação das enzimas proteolíticas. Ocorrem também, com a cocção, alterações estruturais das proteínas, provocando o aumentando da susceptibilidade à hidrólise enzimática. Onde os autores concluíram que o teor de taninos diminuiu com o tempo de armazenamento e com o cozimento, o que pode ser um fator para o aumento da digestibilidade com o tempo de armazenamento, de três para seis meses (Delfino \& Canniatti-Brazaca, 2010). A diminuição no teor de taninos provavelmente ocorre devida à oxidação e à menor solubilidade, decorrentes de seu maior grau de polimerização. Os polifenóis ligados a outros componentes celulares são mais difíceis de serem extraídos, ocorre modificação de suas estruturas e dos componentes a eles ligados, fazendo com que o teor extraído seja menor após períodos de armazenamento dos grãos (Delfino \& Canniatti-Brazaca, 2010). 
As lecitinas presentes em alimentos como as leguminosas, são inativadas após a cocção. Por outro lado, seria inteligente tomar cuidado com leguminosas cruas, especialmente com o amendoim. Assim como sabemos que a cocção é capaz de inativar as lecitinas, já foi demonstrado, em humanos, que é possível identificar lecitinas no sangue após o consumo de amendoim cru. Efeito de diferentes métodos de cozimento em vegetais no conteúdo de oxalato e observaram que a fervura promoveu uma maior redução no teor de oxalato (30-87\%) e foi mais eficaz do que cozinhar (5-53\%) e do que o forneamento (usado apenas para a batata, sem perda de oxalato) (Benevides et al., 2011). No caso do amendoim, as lecitinas presentes podem ser absorvidas e chegar ao sangue, principalmente quando o alimento é ingerido cru. Evitar o oferecimento de leguminosas cruas aos animais pode eliminar a possibilidade das lecitinas chegarem ativas ou intactas à corrente sanguínea e principalmente devido à possibilidade de contaminação por aflatoxina (Benevides et al., 2011).

Plantas cianogênicas, como a mandioca, apresentam compostos ciânicos e enzimas distribuídas em concentrações variáveis nas suas diferentes partes. Pela ruptura da estrutura celular da raiz, as enzimas presentes (linamarase; $\beta$-glicosidase), degradam estes compostos, liberando o ácido cianídrico (HCN), que é o princípio tóxico da mandioca e cuja ingestão ou ainda a inalação, representa sério perigo à saúde, podendo advir sintomas de intoxicação a depender da quantidade e tipo de alimento ingerido, podendo ocorrer casos extremos de envenenamento (Benevides et al., 2011). Avaliar-se que o consumo de alimento contendo o $\mathrm{HCN}$, em uma concentração entre 0,5 a 3,5 $\mathrm{mg}$ de ácido cianídrico por kg de peso vivo, possa levar o indivíduo à morte em poucos minutos. Entretanto, o risco de intoxicação pode ser minimizado a partir da utilização de processos de preparação, tais como: cozimento, fritura e secagem, que reduzem o teor do composto no alimento. Esse efeito benéfico é resultante da remoção de glicosídeos cianogênicos, da inativação das $\beta$-glicosidases, ou de ambos Helbig et al. (2008). Para a alimentação animal, o descanso da casca da mandioca ao ar livre por $24 \mathrm{~h}$ é suficiente para a dissipação do $\mathrm{HCN}$ e uso da casca na dieta de ruminantes.

O conteúdo de gossipol livre nas sementes íntegras de algodão é de cerca de $0,5 \%$, nos processos mecânicos de extração envolvendo pressão e tratamento térmico estes níveis reduzem para cerca de 0,05\% (Gadelha et al., 2011).

A cocção de alimentos para a alimentação animal, na tentativa de diminuir ou viabilizar os efeitos anti-nutricionais pode ser feita de forma industrial e em larga escala, em processos "comuns" dentro das indústrias produtoras de ração, como a peletização, laminação e extrusão, já largamente empregados atualmente.

\section{Peletização}

A peletização consiste um processo utilizado pela indústria de rações para melhorar o desempenho dos animais, e pode ser definida como a aglomeração de partículas (pequenas) de um ingrediente ou de uma mistura de ingredientes, por meio de processos mecânicos (moagem), em combinação com umidade, pressão e calor, fazendo com que ocorram mudanças na estrutura dos alimentos. Na literatura, as principais vantagens da peletização são de melhorar a digestibilidade de nutrientes, aumentar o consumo de ração, reduzir o gasto de energia de mantença, diminuir o desperdício da ração e reduzir a contaminação por microrganismos na ração. Para que ocorra a peletização, ocorre a mistura de ingredientes previamente moídos. E durante o processamento até a confecção do pelete propriamente dito, a mistura passa por diversos processos, como no condicionador, onde a mistura entra em contato com um vapor entre 70 e $90^{\circ} \mathrm{C}$, contendo $18 \%$ de umidade para facilitar a compactação. O vapor deve penetrar no alimento com a umidade necessária para hidratar, e assim permitir a transferência de calor para produzir um bom peletes. O tempo no condicionador pode variar de 9 segundos até 3 minutos, variando de acordo com a fórmula a ser peletizada. Além disso, a ração peletizada finaliza o processo com uma temperatura entre 75 a $93{ }^{\circ} \mathrm{C}$ devido aos efeitos combinados da adição de vapor, durante o condicionamento, e a fricção do produto com o anel (Prado et al., 1996; Silva et al., 2007). Tal temperatura agindo sobre os alimentos é capaz de inativar diversos fatores anti-nutricionais, a depender da concentração do mesmo no alimento e do tempo que a mistura ficar exposto as referidas temperaturas. Com a elevação da temperatura os polifenóis ou taninos podem: ligar-se com algumas proteínas, serem eliminados na água de cozimento, permanecer livres, ou sofrer polimerização (Silva \& Silva, 1999). 


\section{Extrusão}

A extrusão pode ser definida como uma etapa de processamento industrial de matéria-prima sólida, a qual junta num único equipamento vária operações unitárias e modificações físico-químicas, frequentemente em combinação, como mistura, cozimento, batedura, corte, moldagem, gelatinização, fusão, torra, caramelização, secagem e esterilização e processos como a texturização, culminando na saída do respetivo produto pelos orifícios (Souza \& Andrade, 2000). O processo de extrusão acontece pela utilização de um pistão, de um conjunto de rolos ou de parafuso(s) para forçar o material, normalmente através de uma abertura estreita, a chegar à forma desejada (Lopes-da-Silva et al., 2016). Em muitos processos de extrusão de alimentos, o aquecimento $\left(>100^{\circ} \mathrm{C}\right)$ e a cocção das matérias-primas ocorrem simultaneamente, à medida que estas são misturadas e dão forma ao produto final. Assim, após as respetivas matérias-primas sofrerem um aquecimento que conduz ao seu amolecimento ou até à fusão, o produto é moldado por este orifício de saída, consolidando a sua forma (Lopes-da-Silva et al., 2016). Comparado com os métodos tradicionais de processamento, a extrusão é um tratamento termo-mecânico combinado (que envolve o controle cuidadoso das condições do processo da composição do alimento e teor de umidade, a temperatura de cozimento ao longo da extrusora e a velocidade do parafuso e do canhão), é uma maneira mais rápida e mais consistente para causar a quebra térmica / química de compostos anti-nutricionais e ao mesmo tempo poderiam alterar a natureza física, química e nutricional dos nutrientes, maneiras desejáveis (Nikmaram et al., 2018). As vantagens atribuídas ao processo de extrusão são grandes e diversas, tanto em produtos para consumo humano como animal, como: produtos de confeitarias, massas, cereais matinais, produtos para bebes, sopas desidratadas e proteínas texturizadas. Além disso, o alimento extrusado tem maior qualidade e durabilidade, ocupa menor espaço para armazenamento, tem maior eficiência energética quando comparada a outros processos (Lopes-daSilva et al., 2016).

\section{Gossipol}

Alguns métodos podem ser empregados para mitigar os efeitos do gossipol. Como a adição de outro ingrediente na ração como a lisina. De acordo com Valadares Filho (2006) os teores de lisina disponível se relacionam com os de gossipol livre e são influenciados pelo método de extração do óleo, quando o gossipol livre complexa-se com a lisina seus efeitos tóxicos são abrandados. A ação toxica do complexo ferro-gossipol pode ser reduzida quando se adiciona sais de ferro à dieta, que ao se complexarem com o gossipol, o torna menos danoso aos animais (Nagalakshmi et al., 2007). Xiong et al. (2016) afirmou que a fermentação do farelo de algodão com Candida utilis resultou em queda nos níveis de gossipol livre. Processos como a peletização e extrusão do farelo de algodão, que usam calor úmido no processo, provoca modificações nas propriedades do composto tóxico, diminuindo seus efeitos; porém, esses processos podem diminuir o valor biológico da proteína (Lopes-da-Silva et al., 2016).

O melhoramento genético também tem um importante papel. Em 1959, nos Estados Unidos, uma variedade mutante de algodoeiro com sementes desprovidas de glândulas produtoras de gossipol. A partir daí foram selecionados estes genes para o desenvolvimento de uma cultivar denominada "glandles" (McMichael, 1960)

Em aves, o excesso de gossipol na dieta também pode trazer sérios problemas, como: perda de apetite, depressão da atividade respiratória, anemia, edemas pulmonares, hipertrofia do fígado e necrose muscular cardíaca (Barbosa \& Gattás, 2004). O principal problema do gossipol na avicultura de postura é na coloração da gema, problema esse que ocorre principalmente no período de estocagem dos ovos (Ryan et al., 1986). Attanayaka et al. (2016) observaram que aves alimentadas com maiores teores farelo de semente de algodão apresentaram menores valores de ganho de peso e de carcaça. Isso pode ter ocorrido por maiores teores de fibra e gossipol na ração. O conteúdo de gossipol livre nas sementes íntegras de algodão é de cerca de $0,5 \%$, nos processos mecânicos de extração envolvendo pressão e tratamento térmico estes níveis reduzem para cerca de $0,05 \%$ (Gadelha et al., 2011).

\section{Lignina}

A qualidade da forragem pode ser determinada através de uma análise proposta por Van Soest que divide a planta em duas porções: conteúdo celular (composta basicamente por proteínas, lipídeos, pectina e CHO solúveis que são os açúcares) e parede celular (composta por hemicelulose, celulose, 
lignina, minerais e nitrogênio ligada à lignina). De acordo com essa proposta, temos a fibra solúvel em detergente neutro (FDN) que é composta pela hemicelulose, celulose, lignina e a fibra solúvel em detergente ácido (FDA) que é composta pela celulose e pela lignina (Berchielli et al., 2011; Van Soest, 1994). Uma importante substância que compõe a parede celular é a lignina, que tem o papel de enrijecer a parede celular, porém, limita a disponibilidade dos carboidratos da parede celular aos microrganismos (Van Soest, 1994). Cabe destacar que sua formação começa com o espessamento da parede celular secundária, sendo assim, seu conteúdo aumenta de acordo com o desenvolvimento do vegetal (Lima Júnior et al., 2010). Contudo, por ser um componente da planta e sua concentração vai depender do estágio fisiológico da mesma, uma forma de diminuir o teor de lignina seria o retardamento no crescimento da planta, melhorando a qualidade com o aumento da razão folha/caule e a maior digestibilidade das frações folha e caule (Berchielli et al., 2011).

\section{Estratégias animais para a diminuição dos efeitos dos fatores anti-nutricionais}

Os animais superiores possuem apenas cerca de 30 enzimas ativadas pela presença de compostos tóxicos, que funcionam para torná-los prontamente excretáveis ou farmacologicamente menos ativos. Entretanto, alguns animais desenvolveram um tipo de relação simbiótica mutualística com microrganismos albergados em seus compartimentos gástricos (Hobson \& Stewart, 2012). Essa relação ecológica, evoluindo por 70 milhões de anos, permitiu a "soma" do aparato enzimático de dezenas de gêneros de microrganismos com as enzimas autóctones do hospedeiro (Hobson \& Stewart, 2012; Lima Júnior et al., 2010). Deste modo, a existência de microrganismos no rúmen, capazes de neutralizar compostos tóxicos é, portanto, uma grande vantagem competitiva para os ruminantes, quando comparados com outros herbívoros. Significa dizer que um ruminante, num ambiente selvagem, quando as fontes de alimento são, em geral, limitadas, pode-se valer de uma dieta mais variada ou mesmo ter maiores chances de sobrevivência, por causa da detoxificação microbiana pré-gástrica de plantas que sejam venenosas para competidores, os herbívoros não ruminantes (Hobson \& Stewart, 2012; Lima Júnior et al., 2010).

Teorias ecológicas preveem que mamíferos herbívoros que consomem maiores quantidades de compostos secundários de plantas, perdem menos massa corporal, apresentam menores sinais de toxicidade e mantém um maior balanço energético positivo em sua planta preferida. Especialistas também usam de mecanismos de desintoxicação menos caros e eliminam compostos secundários das plantas do corpo de forma mais eficiente do que os generalistas. No caso de taninos, é possível que a sua composição estrutural diversificada e/ou interações entre diferentes compostos secundários tornem difícil para provar esta teoria ecológica. No entanto, algumas evidências de mecanismo de resposta adaptativa foi encontrado em ovinos e caprinos com experiência em pastejo (Alonso-Díaz et al., 2010; Berchielli et al., 2011; Hobson \& Stewart, 2012).

Provavelmente, a primeira linha de defesa em algumas espécies de animais contra compostos fenólicos ou taninos na dieta (Alonso-Díaz et al., 2010; Mueller-Harvey, 2006) são as proteínas salivares, estas atuam protegendo os animais contra os efeitos pós-ingestivos negativos dos taninos e parecem reduzir o evitado sabor amargo ou as propriedades adstringentes do tanino (Monteiro et al., 2005; Silva \& Silva, 1999). Proteínas salivares ligadas ao tanino incluem proteínas ricas em prolina (PRP) e histatinas (HTS), que têm uma forte afinidade com taninos. Além disso, é importante perceber que mesmo dentro de raças, a resposta dos animais as proteínas salivares ligadas aos taninos podem ser influenciadas pela sua experiência ou grau de especialização/adaptação a um nicho ecológico especial. Por exemplo, animais pastejando uma vegetação rica em taninos pode desenvolver diversos mecanismos para atenuar os efeitos adversos dessas plantas e aumentar a sua ingestão. Vários autores sugerem que a adaptação fisiológica a metabólitos secundários de plantas é uma resposta induzida a sua presença na alimentação (Alonso-Díaz et al., 2010; Hobson \& Stewart, 2012).

As glândulas parótidas produzem mais saliva. Herbívoros, incluindo caprinos, têm glândulas parótidas maiores em relação ao peso vivo e a saliva de alguns destes contém altas concentrações de PRP, em relação aos herbívoros que pastam (Waghorn, 2008), dependendo da espécie animal, e ocorrem, por exemplo, na saliva de humanos, porcos (Sus scrofa L.), ratos, coelhos (Oryctolagus cuniculus L.), cabras, veados e ursos (Ursus spp.), mas a saliva de hamsters (Mesocricetus auratus Waterhouse), ovelhas ou vacas contém poucos ou nenhum PRPs. Como resultado, os ratos são mais 
resistentes aos taninos que hamsters e veados e cabras são mais resistentes que as ovelhas e vacas (Mueller-Harvey, 2006). Deste modo, menos proteína é necessária para ligar todos os taninos, resultando em uma economia quantitativa de nitrogênio, além disso, as PRPs tornam-se ricas em aminoácidos não essenciais e são excretadas em vez dos aminoácidos essenciais da proteína dietética, proporcionando desta vez uma economia qualitativa de nitrogênio (Makkar, 2003).

\section{Considerações finais}

Diversos efeitos negativos são atribuídos aos compostos secundários em alguns vegetais, tais como: redução na biodisponibilidade de minerais (oxalatos, fitatos), redução na digestibilidade de proteínas (taninos), inibição da citocromo oxidase (cianeto), formação de substâncias carcinogênicas (nitritos) e inibição de enzimas proteolíticas (inibidores de proteases). Contudo os tratamentos químicos e/ou físicos são medidas de minimizar os efeitos dessas substâncias no organismo dos animais, processamento, como a maceração, uso da atmosfera controlada, tratamento térmico, trituração, descortiçamento de grãos, tratamento enzimático, alta pressão isostática, dentre outros, poderá eliminr os fatores anti-nutricionais.

Melhoramento genético também já é uma realidade no desenvolvimento de variedades de vegetais com menor quantidade de determinados anti-nutrientes. Sugere-se que os estudos continuem a serem realizados, principalmente, em alimentos de elevado consumo regional, pois, na maioria das vezes, não se têm informações sobre a presença dos compostos secundários em sua composição.

\section{Referências bibliográficas}

Alonso-Díaz, M. A., Torres-Acosta, J. F. J., Sandoval-Castro, C. A. \& Hoste, H. (2010). Tannins in tropical tree fodders fed to small ruminants: a friendly foe?. Small Ruminant Research, 89(2-3):164173. doi: https://doi.org/10.1016/j.smallrumres.2009.12.040

Attanayaka, P. M. G. S. K., Pathirana, A. P. D. G., Priyankarage, N., Silva, S. S. P. \& Nayananjalie, W. A. D. (2016). Effect of substitution of soybean meal with cottonseed meal on the performances of broiler chicken. Journal of Livestock Research, 6(3):24-30. doi: https://doi.org/10.5455/ijlr.20160306102626

Barbosa, F. F. \& Gattás, G. (2004). Farelo de algodão na alimentação de suínos e aves. Revista Eletrônica Nutrime, 1147-156.

Benevides, C. M. J., Souza, M. V., Souza, R. D. B. \& Lopes, M. V. (2015). Fatores antinutricionais em alimentos: revisão. Segurança Alimentar e Nutricional, 18(2):67-79. doi:https://doi.org/10.20396/san.v18i2.8634679

Berchielli, T. T., Pires, A. V. \& Oliveira, S. G. (2011). Nutrição de Ruminantes. Jaboticabal, Brazil: FUNEP.

Delfino, R. R. \& Canniatti-Brazaca, S. G. (2010). Interação de polifenóis e proteínas e o efeito na digestibilidade protéica de feijão comum (Phaseolus vulgaris L.) cultivar Pérola. Ciência e Tecnologia de Alimentos, 30(2):308-312. doi: https://doi.org/10.1590/s0101-20612010000200003

Francis, G., Makkar, H. P. S. \& Becker, K. (2001). Antinutritional factors present in plant-derived alternate fish feed ingredients and their effects in fish. Aquaculture, 199(3-4):197-227. doi: https://doi.org/10.1016/s0044-8486(01)00526-9

Gadelha, I. C. N., Rangel, A. H. N., Silva, A. R. \& Soto-Blanco, B. (2011). Efeitos do gossipol na reprodução animal. Acta Veterinaria Brasilica, 5(2):129-135.

Harborne, J. B. (1999). An overview of antinutritional factors in higher plants. In J. C. Caygill \& H. Mueller (Eds.), Secondary plants products. Antinutritional and beneficial actions in animal feeding (pp. 7-16). Nottingham Univ Press, UK.doi: https://doi.org/10.1142/97898128172730001

Helbig, E., Buchweitz, M. R. D. \& Gigante, D. P. (2008). Análise dos teores de ácidos cianídrico e fítico em suplemento alimentar: multimistura. Revista de Nutrição, 21(3):323-328. doi: https://doi.org/10.1590/s1415-52732008000300007

Hobson, P. N. \& Stewart, C. S. (2012). Rumen Microbial Ecosystem (2 ed.). Londo, UK: Blackie Academic \& Professional. 
Kumar, A. K. \& Sharma, S. (2017). Recent updates on different methods of pretreatment of lignocellulosic feedstocks: a review. Bioresources and Bioprocessing, 4(7):1-19. doi: https://doi.org/10.1186/s40643-017-0137-9

Lima Júnior, D. M., Monteiro, P. d. B. S., Rangel, A. H. N., Maciel, M. V., Oliveira, S. E. O. \& Freire, D. A. (2010). Fatores anti-nutricionais para ruminantes. Acta Veterinaria Brasilica, 4(3):132-143. doi: https://doi.org/10.21708/avb

Lopes-da-Silva, M. F., Santos, L. \& Choupina, A. (2016). A extrusão em tecnologia alimentar: aplicações, caraterísticas dos produtos, composição e tendências futuras. Revista de Ciências Agrárias, 39(1):4-14. doi: https://doi.org/10.19084/rca14103

Makkar, H. P. S. (2003). Effects and fate of tannins in ruminant animals, adaptation to tannins, and strategies to overcome detrimental effects of feeding tannin-rich feeds. Small Ruminant Research, 49(3):241-256. doi: https://doi.org/10.1016/s0921-4488(03)00142-1

McMichael, S. C. (1960). Combined effects of glandless genes gl2 and gl3 on pigment glands in the cotton plant. Agronomy Journal, 52(7):385-386. doi: https://doi.org/10.2134/agronj1960.00021962005200070005x

Monteiro, J. M., Albuquerque, U. P., Araujo, E. \& Amorim, E. L. C. (2005). Taninos: uma abordagem da química à ecologia. Química Nova, 28(5):892-896. doi: https://doi.org/10.1590/s0100$\underline{40422005000500029}$

Mueller-Harvey, I. (2006). Unravelling the conundrum of tannins in animal nutrition and health. Journal of the Science of Food and Agriculture, 86(13):2010-2037. doi: https://doi.org/10.1002/jsfa.2577

Nagalakshmi, D., Rao, S. V. R., Panda, A. K. \& Sastry, V. R. B. (2007). Cottonseed meal in poultry diets: a review. The Journal of Poultry Science, 44(2):119-134. doi: https://doi.org/10.3382/ps.0290486

Namdeo, A. G. (2007). Plant cell elicitation for production of secondary metabolites: a review. Pharmacognosy Reviews, 1(1):69-79.

Nikmaram, N., Budaraju, S., Barba, F. J., Lorenzo, J. M., Cox, R. B., Mallikarjunan, K. \& Roohinejad, S. (2018). Application of plant extracts to improve the shelf-life, nutritional and health-related properties of ready-to-eat meat products. Meat Science, 145245-255. doi: https://doi.org/10.1016/j.meatsci.2018.06.031

Owens, F. 1. N., Zinn, R. A. \& Kim, Y. K. (1986). Limits to starch digestion in the ruminant small intestine. Journal of Animal Science, 63(5):1634-1648. doi: https://doi.org/10.2527/jas1986.6351634x

Prado, I. N., Pinto, A. A., Zeoula, L. M. \& Martins, A. S. (1996). Farelo de canola farelado, moído e peletizado sobre o desempenho e características de carcaça de novilhas Nelore. Revista UNIMAR, 18(3):567-575.

Reis, W. d. (2006). Degradabilidade de grãos secos e ensilados de híbridos de milho submetidos a formas de processamento. $\mathrm{PhD}$, Universidade de São Paulo, Botucatu, São Paulo, Brasil.

Ryan, J. R., Kratzer, F. H., Grau, C. R. \& Vohra, P. (1986). Glandless cottonseed meal for laying and breeding hens and broiler chicks. Poultry Science, 65(5):949-955.

Silva, M. R. \& Silva, M. A. A. P. d. (1999). Aspectos nutricionais de fitatos e taninos. Revista de Nutrição, 1221-32. doi: https://doi.org/10.1590/s1415-52731999000100002

Silva, R. R., Silva, F. F., Prado, I. N., Carvalho, G. G. P., Oliveira, A. P., Chaves, M. A. \& Pinnheiro, A. A. (2007). Efeito da ração farelada e peletizada sobre o comportamento ingestivo de bezerros Holandeses. Archivos de Zootecnia, 56(214):227-238.

Souza, C. G., Moraes, J. P. G., Costa, C., Meirelles, P. R. L., Reis, W., Gonçalves, H. C. \& Factori, M. A. (2010). Degradabilidade de grãos secos e ensilados de sorgo, com e sem tanino, submetidos à granulometrias. Boletim da Indústria Animal, 67163-174.

Souza, R. \& Andrade, C. (2000). Investigação dos processos de gelatinização e extrusão de amido de milho. Polímeros: Ciência e Tecnologia, 10(1):24-30. doi: https://doi.org/10.1590/s0104$\underline{14282000000100006}$ 
Valadares Filho, S. C. (2006). Tabelas brasileiras de composição de alimentos para bovinos (Vol. 1). Viçosa: UFV.

Van Soest, P. J. (1994). Nutritional ecology of the ruminant (Vol. 1). Ithaca, NY, USA: Cornell University Press.

Waghorn, G. (2008). Beneficial and detrimental effects of dietary condensed tannins for sustainable sheep and goat production-Progress and challenges. Animal Feed Science and Technology, 147(13):116-139. doi: https://doi.org/10.1016/j.anifeedsci.2007.09.013

Xiong, J. L., Wang, Z. J., Miao, L. H., Meng, F. T. \& Wu, L. Y. (2016). Growth performance and toxic response of broilers fed diets containing fermented or unfermented cottonseed meal. Journal of Animal Feed Science, 25(4):348-353. doi: https://doi.org/10.2527/jam2016-0949

Recebido: 23 de março, 2019.

Aprovado: 6 de maio, 2019.

Publicado: 27 de maio, 2019.

Licenciamento: Este artigo é publicado na modalidade Acesso Aberto sob a licença Creative Commons Atribuição 4.0 (CC-BY 4.0), a qual permite uso irrestrito, distribuição, reprodução em qualquer meio, desde que o autor e a fonte sejam devidamente creditados. 